\title{
A Metastatic Cervical Adenocarcinoma Patient Carrying HER2 G292R Achieved Complete Response Upon Pyrotinib Treatment
}

\author{
Jing Liu',* \\ Yanhong Zhuo ${ }^{2, *}$ \\ Feng Wang ${ }^{3, *}$ \\ Zirong $\mathrm{Li}^{4, *}$ \\ Yibin Lin' \\ $\mathrm{Li} \mathrm{Li}^{\mathrm{l}}$ \\ Junping $\operatorname{Pan}^{5}$ \\ Yanwen Song ${ }^{5}$ \\ Haiwei Du ${ }^{6}$ \\ Chanhe $\mathrm{Li}^{7}$ \\ Qin $X u^{\prime}$
}

'Department of Gynecology, Fujian Medical University Cancer Hospital \& Fujian Cancer Hospital, Fuzhou, People's Republic of China; ${ }^{2}$ Department of Radiotherapy, Zhangzhou Hospital \& Teaching Hospital of Fujian Medical University, Zhangzhou, People's Republic of China; ${ }^{3}$ Department of Outpatient, Fujian Hospital of People's Armed Police, Fuzhou, People's Republic of China; ${ }^{4}$ Department of Radiation

Oncology, Longyan First Hospital Affiliated to Fujian Medical University, Longyan, People's Republic of China; ${ }^{5}$ Department of Radiotherapy, Fujian Medical University Cancer Hospital \& Fujian Cancer Hospital, Fuzhou, People's Republic of China; ${ }^{6}$ Department of Data Science, Burning Rock Biotech, Guangzhou, People's Republic of China; ${ }^{7}$ Department of Medicine, Burning Rock Biotech, Guangzhou, People's Republic of China

*These authors contributed equally to this work

Correspondence: Qin Xu

Department of Gynecology, Fujian

Medical University Cancer Hospital \&

Fujian Cancer Hospital, No. 420 Fuma

Road, Fuzhou, 350014, Fujian, People's

Republic of China

Email xuqin772836@163.com

\begin{abstract}
Cervical cancer patients who develop distant metastasis are rarely curable with very limited treatment options. Chemotherapy is often administered but with limited efficacy. Immunotherapy and anti-angiogenesis therapy are recommended for selected cases of recurrent or metastatic cervical cancers. The clinical efficacy of inhibitors targeting HER2, a commonly mutated gene in cervical cancer, has not been elucidated. Herein, we report a metastatic cervical adenocarcinoma patient carrying HER2 G292R who benefited from pyrotinib after progression on radio-chemotherapy, achieving complete response (CR) with a progression-free survival of 25 months and counting. Our study sheds light on the treatment options for previously treated metastatic cervical adenocarcinoma patients harboring activating HER2 mutations.
\end{abstract}

Keywords: cervical cancer, adenocarcinoma, HER2, pyrotinib, complete response

\section{Introduction}

Cervical cancer is one of the leading causes of morbidity and cancer deaths in women worldwide and in China. ${ }^{1,2}$ Local radiotherapy, chemotherapy or the combination of chemotherapy and bevacizumab are the standard first-line treatment for recurrent and metastatic cervical cancer, ${ }^{3}$ but the current options for second-line and beyond treatment of cervical cancer remain limited.

$H E R 2$ (ERBB2) encodes the epidermal growth factor family member of receptor tyrosine kinases (RTKs). Somatic HER2 alterations including HER2 single nucleotide variants and $H E R 2$ amplification, occurring in $14 \%$ of cervical cancers, are considered to be oncogenic and associated with poor prognosis. ${ }^{4}$ Pyrotinib, an irreversible panHER tyrosine kinase inhibitor against EGFR, HER2 and HER4, combined with capecitabine has been approved by China National Medical Products Administration for the treatment of patients with HER2-overexpressing advanced or metastatic breast cancer who have been previously treated with anthracycline or taxane chemotherapy. ${ }^{5}$ Whether patients with HER2-mutant cervical cancer benefit from pyrotinib treatment has not been documented. In this study, we report the first clinical evidence of the efficacy of pyrotinib in a poorly differentiated metastatic cervical adenocarcinoma patient carrying HER2 G292R who progressed on radio-chemotherapy.

\section{Case Presentation}

A woman in her 40s presented with irregular vaginal bleeding and was referred to our clinic in May 2018. The patient's treatment history is shown in Figure 1. She was 


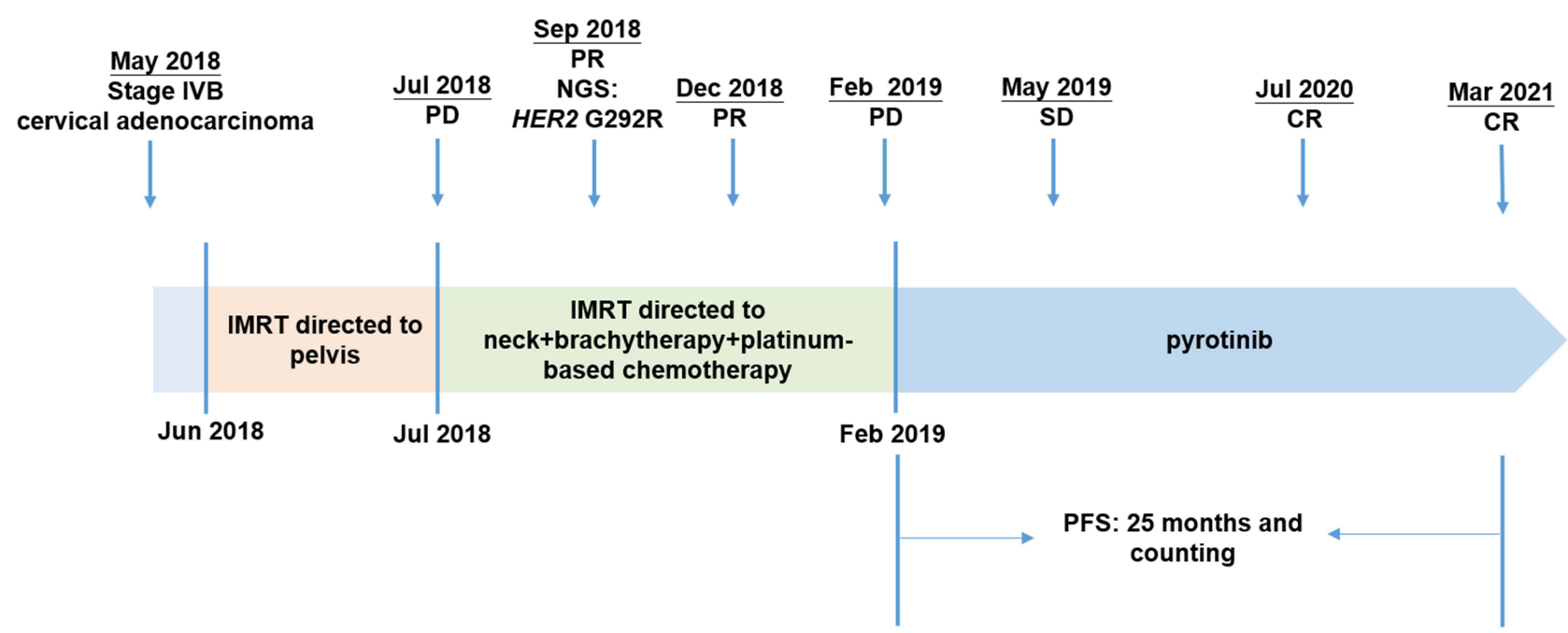

Figure I A summary of patient's treatment history.

Abbreviations: PR, partial response; PD, progressive disease; SD, stable disease; CR, complete response; PFS, progression-free survival; NGS, next-generation sequencing; HER2, ERBB2 receptor tyrosine kinase 2; IMRT, intensity-modulated radiation therapy.

diagnosed with stage IVB cervical adenocarcinoma (the red arrow in Figure 2A) with metastases to parametrial lymph node, retroperitoneal lymph node, pelvic lymph node, and neck lymph node. Hematoxylin \& eosin (H\&E) staining showed a primary cervical carcinoma (Figure 3A) and the metastases to neck lymph node (Figure 3B). Immunohistochemistry staining was performed on the primary cervical tumor and demonstrated that $1 \%$ of tumor cells were positive for HER2 overexpression (Figure 3C). The patient refused to receive chemotherapy at the beginning of treatment and instead received intensity-modulated radiation therapy (IMRT) on pelvis. Unfortunately, she developed metastases to liver (the red arrow in Figure 2B) and left supraclavicular lymph node two weeks later. Under the consent of the patient, IMRT directed to neck in combination with brachytherapy and platinum-based chemotherapy (paclitaxel and nedaplatin) were administered in July 2018. The pelvic magnetic resonance imaging (MRI), abdominal MRI, and chest computed tomography (CT) scans in September 2018 demonstrated partial response (PR) in parametrial lymph node, retroperitoneal lymph node, pelvic lymph node, and metastatic liver tumor (the red arrow in Figure 2C). Re-examination in December 2018 demonstrated the complete remission of the primary cervical tumor. The
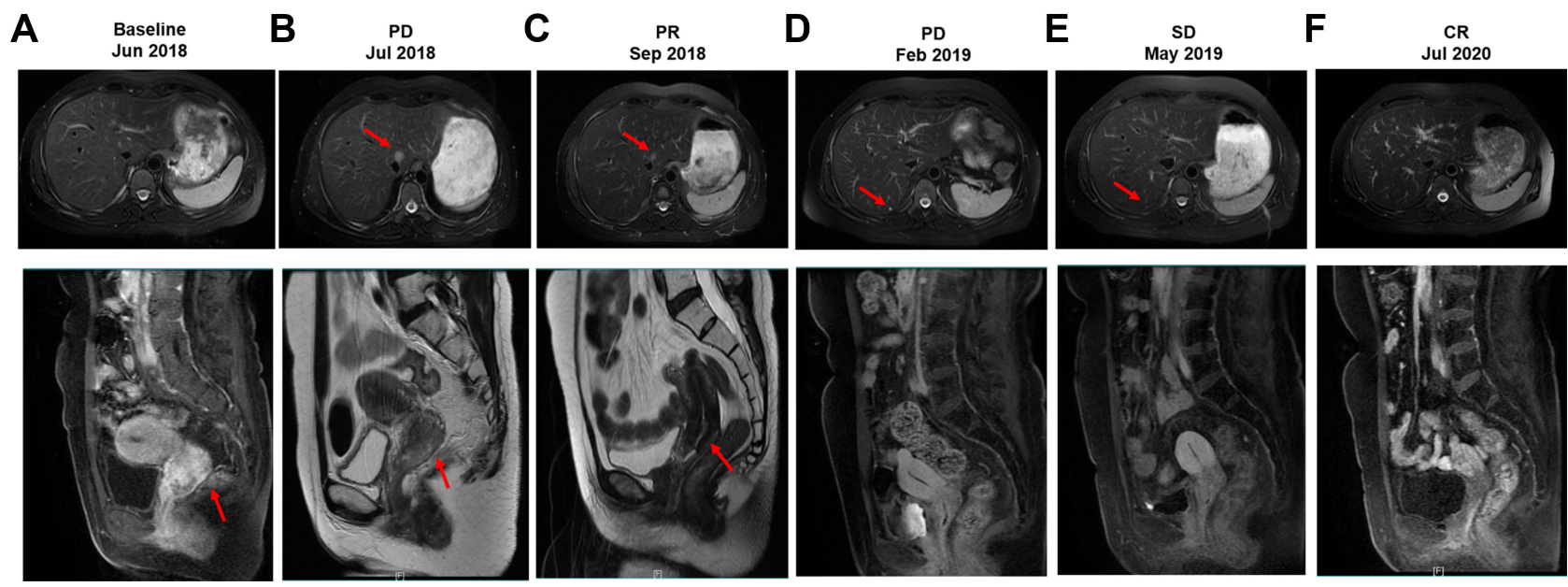

Figure 2 Magnetic resonance imaging (MRI) of the liver (upper) and cervical (lower) lesions at treatment milestones. (A) at baseline; (B) two weeks after IMRT directed to pelvis; (C) two months after IMRT directed to neck combined with brachytherapy and platinum-based chemotherapy (PR); (D) seven months after IMRT directed to neck combined with brachytherapy and platinum-based chemotherapy (PD). (E) three months after pyrotinib treatment (SD); (F) seventeen months after pyrotinib treatment (CR). The red arrows in upper and lower panels indicated metastatic live lesion and the primary cervical adenocarcinoma, respectively.

Abbreviations: PR, partial response; PD, progressive disease; SD, stable disease; CR, complete response; IMRT, intensity-modulated radiation therapy 

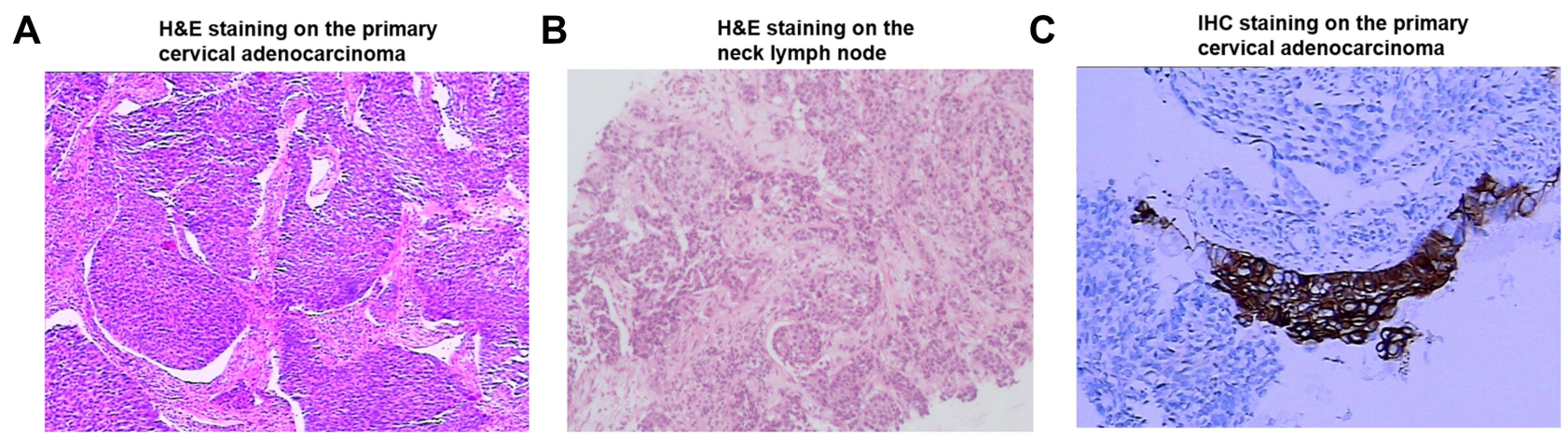

Figure 3 Hematoxylin \& eosin (H\&E)/immunohistochemistry (IHC) staining on the primary cervical adenocarcinoma/neck lymph node. (A) H\&E staining on the primary cervical adenocarcinoma; (B) H\&E staining on the neck lymph node; (C) IHC staining for HER2 on the primary cervical adenocarcinoma. Abbreviations: H\&E, hematoxylin \& eosin; IHC, immunohistochemistry; HER2, ERBB2 receptor tyrosine kinase 2.

tumor assessment remained PR in metastatic liver tumor. In February 2019, the patient experienced disease progression with the newly developed metastatic liver lesion (the red arrow in Figure 2D). The patient was subsequently treated with oral pyrotinib (400 $\mathrm{mg} /$ day) based on the presence of HER2 G292R with an allele frequency of $11.49 \%$, negative HER2 amplification, and microsatellite stable (MSS) in cervical biopsy detected by capture-based targeted sequencing (Burning Rock Biotech, Guangzhou, China) using a panel consisting of 520 cancer-related genes prior to pyrotinib treatment. Due to the intolerant hand-foot syndrome as the pyotinib-related adverse event, the dose of pyrotinib was reduced to $240 \mathrm{mg} / \mathrm{day}$. The patient achieved stable disease in May 2019 (Figure 2E) and the complete response in the metastatic liver tumor (the red arrow in Figure 2F) and metastases to lymph nodes in July 2020. She remained as tumor-free for 8 months and counting at the time of manuscript submission.

\section{Discussion}

To the best of our knowledge, this is the first to demonstrate the clinical efficacy of pyrotinib in a cervical cancer patient harboring HER2 G292R who progressed on radiochemotherapy.

Currently, no HER2 or pan-HER inhibitors have been approved for HER2-mutant cervical cancers, owing to the fact that the clinical efficacy of HER2 or pan-HER inhibitors against HER 2 mutations remains elusive in cervical cancer. HER2 G292R, which affects the extracellular domain of HER2, has been identified in non-small-cell lung cancer, breast cancer, and gallbladder carcinoma. ${ }^{6,7}$ HER2 G292R potentially leads to a gain of function of HER2 protein. In vitro studies have demonstrated that overexpression of HER2
G292R increase gallbladder cancer cell proliferation. ${ }^{6}$ This mutation has not been reported in cervical cancers before.

Pyrotinib has been approved for HER2-positive breast cancer. ${ }^{5}$ Previous studies have demonstrated the efficacy of pyrotinib against HER2-mutant solid tumors. A phase I basket trial completed in the United States has showed that patients with HER2-mutant lung cancer and other solid tumors obtained clinical benefit from pyrotinib treatment with a median progression-free survival (PFS) of 4.4 and 5.3 months, respectively. ${ }^{8}$ A multicenter, open-label Phase II study has demonstrated that pyrotinib showed promising antitumor activity with an objective response rate of $30 \%$ and a PFS of 6.9 months in Chinese patients with HER2mutant lung adenocarcinoma who progressed on platinumbased chemotherapy. ${ }^{9}$ However, patients with cervical cancer or harboring HER2 G292R are not included in the above studies. Several activating HER2 mutations occurring in the extracellular domain of HER2 protein have been identified in solid tumors, including G265K, G292R, G309A/E, S310F, S310Y, and E405D. ${ }^{4,6,10,11}$ HER2 G309A/E, S310F, and E405D mutations have been shown to respond to afatinib or neratinib in vitro or in vivo. ${ }^{4,10}$ A prior study has demonstrated that $5.8 \%$ of cervical tumors carry HER 2 extracellular domain mutations. ${ }^{4}$ The most common subtype of HER2 mutations in cervical cancer is $\mathrm{S} 310 \mathrm{~F} / \mathrm{Y}^{4}$ in contrast to the HER2 exon 20 insertions and V777/L755 missense mutations most commonly found in lung cancer and breast cancer, respectively. Cervical cancers carrying S310F or E405D are sensitive to afatinib or neratinib in vitro. ${ }^{4}$ The SUMMIT basket trial has demonstrated the promising activity of neratinib in patients with breast, cervical, and biliary cancers carrying HER2 mutations. ${ }^{12}$ The updated results from SUMMIT basket trial have demonstrated that 4 of 16 HER2mutant cervical cancer patients derived objective response to 
neratinib with a median PFS of 7.0 months. All responders harbored HER2 S310F mutations, including one who achieved complete response to neratinib. ${ }^{13}$ In this study, the HER2 G292R-positive patient derived complete remission of the primary cervical adenocarcinoma when treated with radio-chemotherapy. The patient subsequently derived complete remission of the metastatic tumors and remained in complete remission of the primary cervical tumor when treated with pyrotinib with a PFS of 25 months and counting. Both previous studies and ours suggest that HER2 extracellular domain mutations might be the promising therapeutic target in cervical cancer. Large cohorts and clinical trials are needed to further evaluate the efficacy of pan-HER inhibitor pyrotinib against HER2 G292R and other HER2 extracellular domain mutations in cervical cancer.

In summary, we revealed the first clinical evidence that a metastatic cervical adenocarcinoma patient carrying HER2 G292R mutation achieved CR with a PFS of 25 months and counting upon pyrotinib treatment. Our data shed light on the treatment options for previously treated advanced cervical adenocarcinoma patients harboring this or other activating HER2 mutations.

\section{Ethics Statement}

This study was approved by the Ethics Committee of Fujian Cancer Hospital. Patient provided informed consent to the study and permitted the use of tumor tissue. Written informed consent was obtained from the patient for publication of this case report.

\section{Funding}

This study was supported by Natural Science Foundation of Fujian Province (No.2020J011126) and Joint Funds for the Innovation of Science and Technology Program of Fujian Province, China (No.2018Y9110).

\section{Disclosure}

The authors have no conflicts of interest to declare.

\section{References}

1. Siegel RL, Miller KD, Jemal A. Cancer statistics, 2020. CA Cancer J Clin. 2020;70(1):7-30. doi:10.3322/caac.21590

2. Chen W, Zheng R, Baade PD, et al. Cancer statistics in China, 2015. CA Cancer J Clin. 2016;66(2):115-132. doi:10.3322/caac.21338

3. NCCN Clinical Practice Guidelines in Oncology. Cervical cancer Version 1.2021. Available from: www.nccn.org. Accessed August 31, 2021.

4. Zammataro L, Lopez S, Bellone S, et al. Whole-exome sequencing of cervical carcinomas identifies activating ERBB2 and PIK3CA mutations as targets for combination therapy. Proc Natl Acad Sci U S A. 2019;116(45):22730-22736. doi:10.1073/pnas.1911385116

5. Blair HA. Pyrotinib: first global approval. Drugs. 2018;78(16): 1751-1755. doi:10.1007/s40265-018-0997-0

6. Li M, Zhang Z, Li X, et al. Whole-exome and targeted gene sequencing of gallbladder carcinoma identifies recurrent mutations in the ErbB pathway. Nat Genet. 2014;46(8):872-876. doi:10.1038/ng.3030

7. Chakravarty D, Gao J, Phillips SM, et al. OncoKB: a precision oncology knowledge base. JCO Precis Oncol. 2017;1:1-6. doi:10. 1200/po.17.00011

8. Li BT, Li T, Johnson ML, et al. Safety and efficacy of pyrotinib in patients with NSCLC and other advanced solid tumors with activating HER2 alterations: a Phase I basket trial. J Clin Oncol. 2020;38 (15_suppl):3510. WCLC Abastrac \#3510. doi:10.1200/JCO.2020. 38.15_suppl.3510

9. Zhou C, Li X, Wang Q, et al. Pyrotinib in HER2-mutant advanced lung adenocarcinoma after platinum-based chemotherapy: a multicenter, open-label, single-arm, Phase II Study. J Clin Oncol. 2020;38(24):2753-2761. doi:10.1200/jco.20.00297

10. Bose R, Kavuri SM, Searleman AC, et al. Activating HER2 mutations in HER2 gene amplification negative breast cancer. Cancer Discov. 2013;3(2):224-237. doi:10.1158/2159-8290.cd-12-0349

11. Greulich H, Kaplan B, Mertins P, et al. Functional analysis of receptor tyrosine kinase mutations in lung cancer identifies oncogenic extracellular domain mutations of ERBB2. Proc Natl Acad Sci U S A. 2012;109(36):14476-14481. doi:10.1073/pnas.1203201109

12. Hyman DM, Piha-Paul SA, Won H, et al. HER kinase inhibition in patients with HER2- and HER3-mutant cancers. Nature. 2018;554 (7691):189-194. doi:10.1038/nature25475

13. Oaknin A, Friedman CF, Roman LD, et al. Neratinib in patients with HER2-mutant, metastatic cervical cancer: findings from the Phase 2 SUMMIT basket trial. Gynecol Oncol. 2020;159(1):150-156. doi:10. 1016/j.ygyno.2020.07.025
OncoTargets and Therapy

\section{Publish your work in this journal}

OncoTargets and Therapy is an international, peer-reviewed, open access journal focusing on the pathological basis of all cancers, potential targets for therapy and treatment protocols employed to improve the management of cancer patients. The journal also focuses on the impact of management programs and new therapeutic agents and protocols on patient perspectives such as quality of life, adherence and satisfaction. The manuscript management system is completely online and includes a very quick and fair peer-review system, which is all easy to use. Visit http://www.dovepress.com/ testimonials.php to read real quotes from published authors. 